\title{
THE ATTITUDE CONTROL SYSTEM OF THE WILKINSON MICROWAVE ANISOTROPY PROBE
}

\author{
F. Landis Markley, ${ }^{*}$ Stephen F. Andrews, $†$ James R. O’Donnell, Jr., ${ }^{* *}$ David K. Ward** \\ NASA Goddard Space Flight Center
}

\begin{abstract}
The Wilkinson Microwave Anisotropy Probe mission produces a map of the cosmic microwave background radiation over the entire celestial sphere by executing a fast spin and a slow precession of its spin axis about the Sun line to obtain a highly interconnected set of measurements. The spacecraft attitude is sensed and controlled using an inertial reference unit, two star trackers, a digital sun sensor, twelve coarse sun sensors, three reaction wheel assemblies, and a propulsion system. Sufficient attitude knowledge is provided to yield instrument pointing to a standard deviation $(1 \sigma)$ of 1.3 arc-minutes per axis. In addition, the spacecraft acquires and holds the sunline at initial acquisition and in the event of a failure, and slews to the proper orbit adjust orientations and to the proper off-sunline attitude to start the compound spin. This paper presents an overview of the design of the attitude control system to carry out this mission and presents some early flight experience.
\end{abstract}

\section{INTRODUCTION}

The Wilkinson Microwave Anisotropy Probe (WMAP), the second Medium-Class Explorer (MDEX) mission, was launched on June 30, 2001 as a follow-on to the Cosmic Background Explorer (COBE), which made precise measurements of the cosmic microwave background

* Aerospace Engineer, Fellow AIAA, Greenbelt, MD, 20771, USA

$\uparrow$ Aerospace Engineer, Greenbelt, MD, 20771, USA

** Aerospace Engineer, Senior Member AIAA, Greenbelt, MD, 20771, USA 
$(C M B)$ that is believed to be a remnant of the Big Bang marking the birth of the universe. ${ }^{1-4}$ WMAP has measured the $\mathrm{CMB}$ anisotropy with sensitivity 50 times that of the Differential Microwave Radiometer (DMR) instrument on COBE and angular resolution 30 times finer, specifically 20 microKelvin and 14 arc minutes, respectively, enabling scientists to determine the values of key cosmological parameters and to answer questions about the origin of structure in the early universe and the fate of the universe., 6

Since the major error sources in the DMR data arose from COBE's low Earth orbit, WMAP was placed in a Lissajous orbit around the Sun-Earth $\mathrm{L}_{2}$ Lagrange point to minimize magnetic, thermal, and radiation disturbances from the Earth and Sun. WMAP attained its Lissajous orbit around $L_{2}$ in early October 2001, about 100 days after launch by a Delta II launch vehicle, using a lunar gravity assist following three phasing loops, as shown in Figure 1. The trajectory design and operations are discussed in detail in Refs. 7-12.

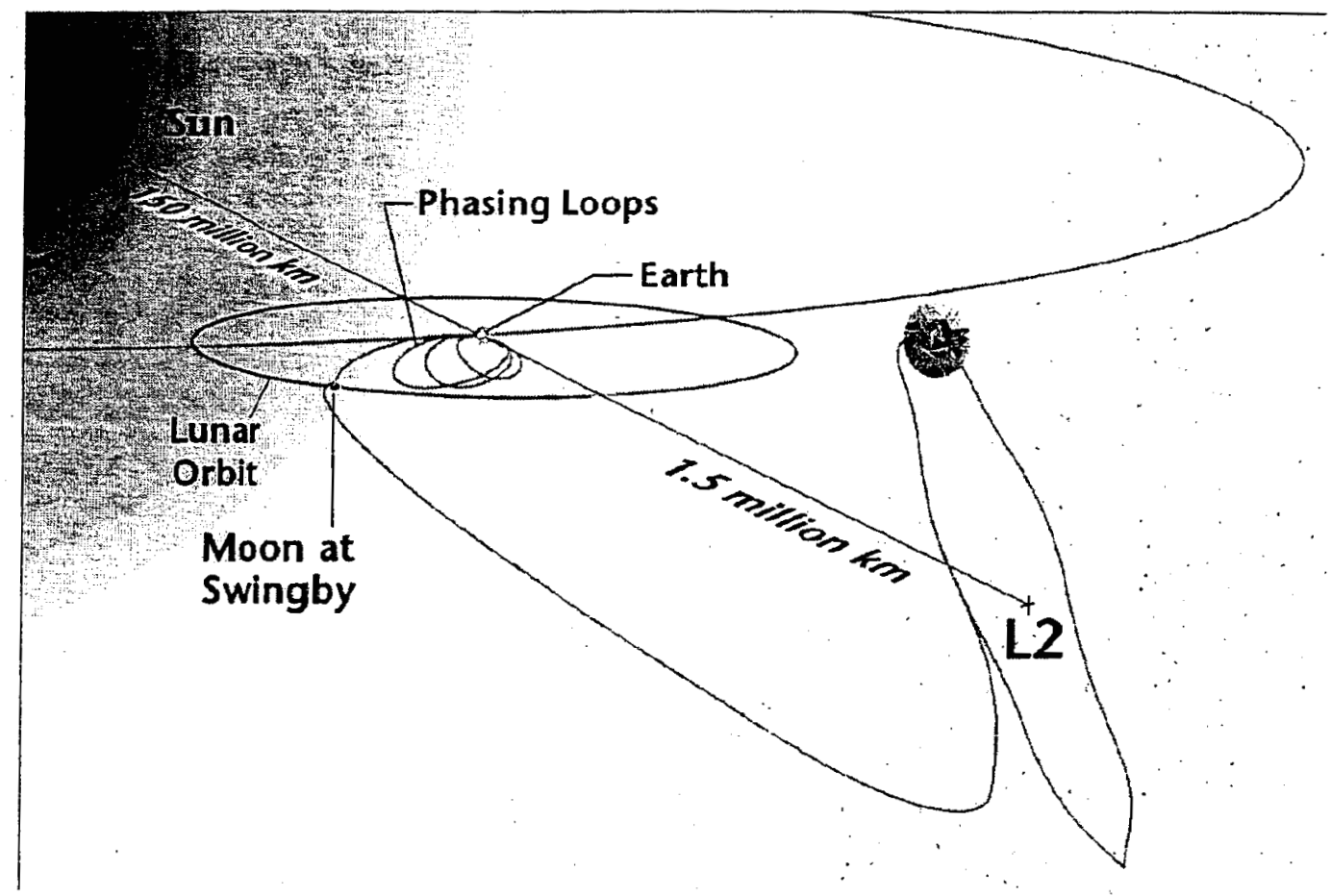

Figure 1: WMAP Trajectory to $\mathrm{L}_{2}$ 
The WMAP instrument includes radiometers at five frequencies, passively cooled to about $90^{\circ} \mathrm{K}$, covering two fields of view (FOVs) $141^{\circ}$ apart on the celestial sphere. The WMAP observatory executes a fast spin coupled with a slower precession of its spin axis at a constant angle of $22.5^{\circ}$ from the Sun line to obtain a highly interconnected set of measurements over an annulus between $87^{\circ}$ and $132^{\circ}$ from the Sun. The rotation about the spin axis has to be at least an order of magnitude faster than the rate of precession of the spin axis; the rates chosen were $2.784 \mathrm{deg} / \mathrm{s}$ $(0.464 \mathrm{rpm})$ for the spin rate and $-0.1 \mathrm{deg} / \mathrm{s}$ ( 1 revolution per hour) for the precession rate. Figure 2 shows the scan pattern covered by one of the two FOVs in one complete spacecraft precession ( 1 hour), displayed in ecliptic coordinates in which the ecliptic equator runs horizontally across the map. The bold circle shows the path for a single spin ( 2.2 minutes). As the Earth revolves around the Sun, this annulus of coverage revolves about the ecliptic pole as shown in Figure 3. Thus the entire celestial sphere will be observed once every six months, or eight times in the planned mission life of four years.

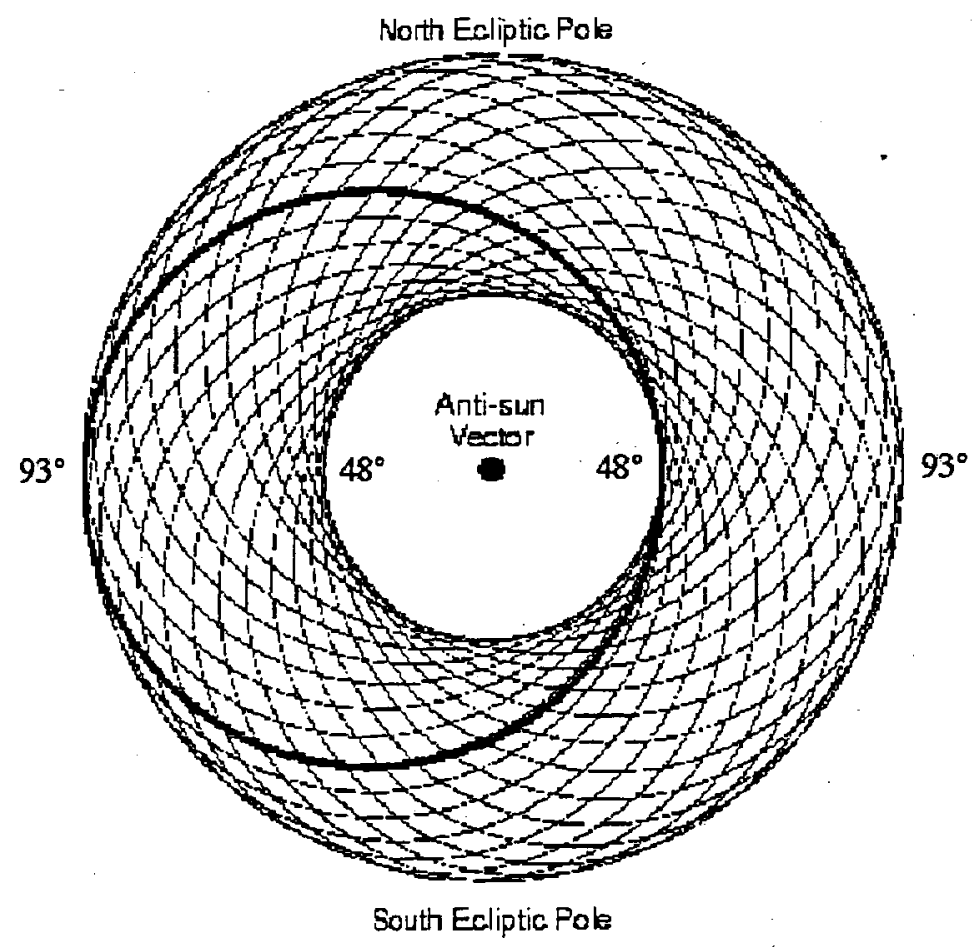

Figure 2: WMAP Scan Pattern 




Figure 3: WMAP Spin-Scan Concept

This paper gives an overview of the Attitude Control System (ACS) that acquires and maintains the spacecraft orbit, controls the spacecraft angular momentum, provides for safety in the event of an anomaly, and implements the spin-scan observing strategy while minimizing thermal and magnetic fluctuations, especially those synchronous with the spin period. More detail can be found in Refs. 13-19.

\section{ACS OVERVIEW}

WMAP uses three right-handed, orthonormal coordinate systems. The Geocentric Inertial frame (GCI) is an Earth-centered frame with its $\mathbf{x}_{I}$ axis pointing to the vernal equinox, its $\mathbf{z}_{\mathbf{I}}$ axis pointing to the North Celestial Pole (parallel to the Earth's spin axis), and $\mathbf{y}_{l}=\mathbf{z}_{\mathrm{I}} \times \mathbf{x}_{\mathrm{I}}$. The Rotating Sun Referenced frame (RSR) is a spacecraft-centered frame in which the $\mathbf{z}_{R}$ axis points from the WMAP spacecraft to the Sun, $\mathbf{x}_{R}$ is a unit vector in the direction of $\mathbf{z}_{R} \times \mathbf{z}_{\mathrm{I}}$, and 
$\mathbf{y}_{R}=\mathbf{z}_{R} \times \mathbf{x}_{R}$. The RSR frame rotates at approximately $1 \%$ day with respect to the GCI frame. The body frame is centered at the spacecraft center of mass with $\mathbf{z}_{\mathrm{B}}$ axis parallel to the spacecraft centerline, directed from the instrument to the solar arrays, $\mathbf{y}_{\mathrm{B}}$ axis normal to the instrument radiator faces, and $\mathbf{x}_{B}=\mathbf{y}_{B} \times \mathbf{z}_{\mathrm{B}}$, as shown in Figure 4. As a minimum, all the WMAP ACS control modes maintain the spacecraft $\mathrm{z}_{\mathrm{B}}$ axis within $25^{\circ}$ of the Sun, to satisfy thermal and power constraints.

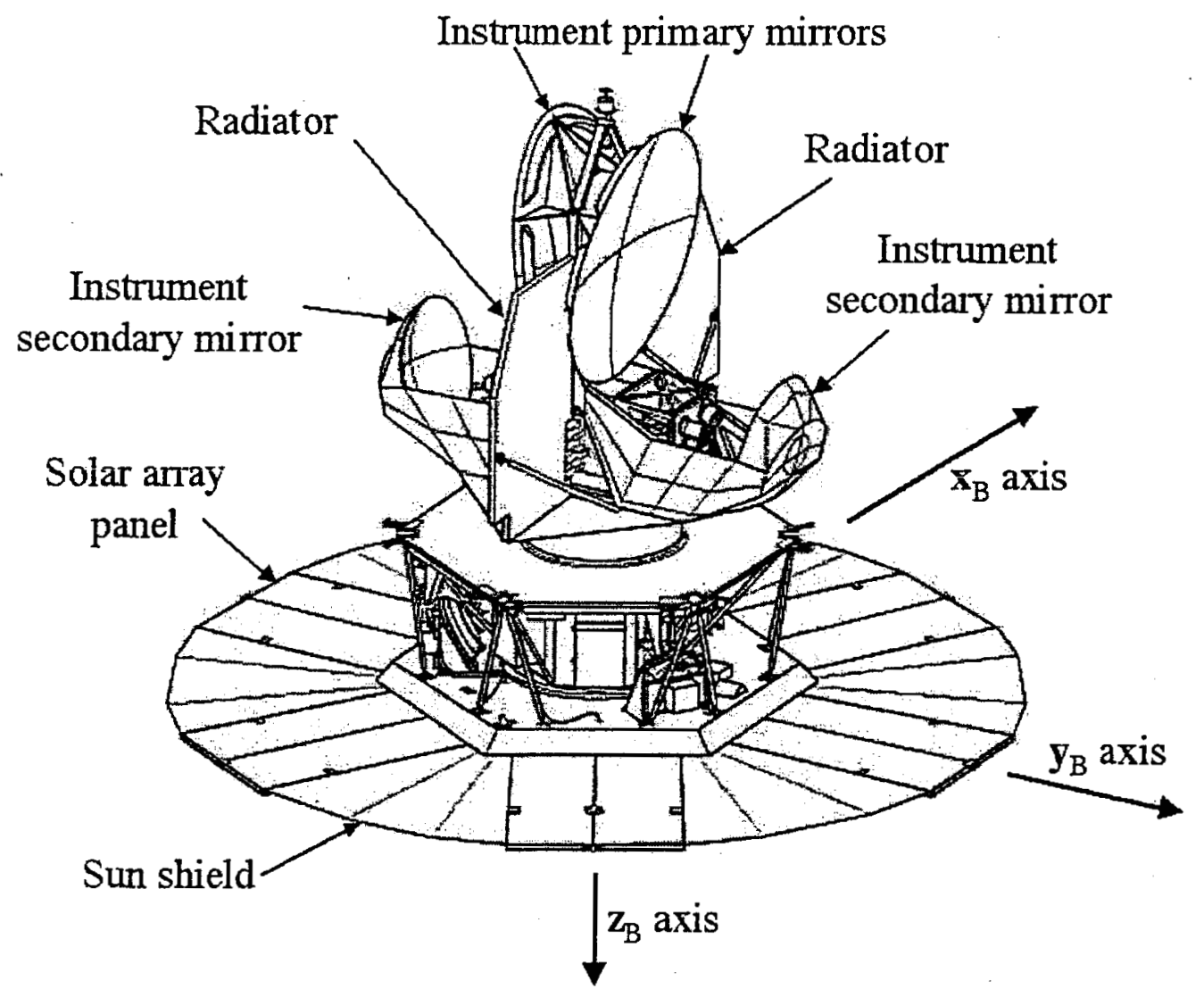

Figure 4: Spacecraft Layout

The WMAP attitude is sensed by an Inertial Reference Unit (IRU); two Autonomous Star Trackers (ASTs), a Digital Sun Sensor (DSS), and twelve Coarse Sun Sensors (CSSs); it is controlled by three Reaction Wheel Assemblies (RWAs) and a propulsion system. Figure 5 illustrates the WMAP ACS architecture. More detail on the WMAP ACS hardware suite can be found in Refs. 16 and 19. 


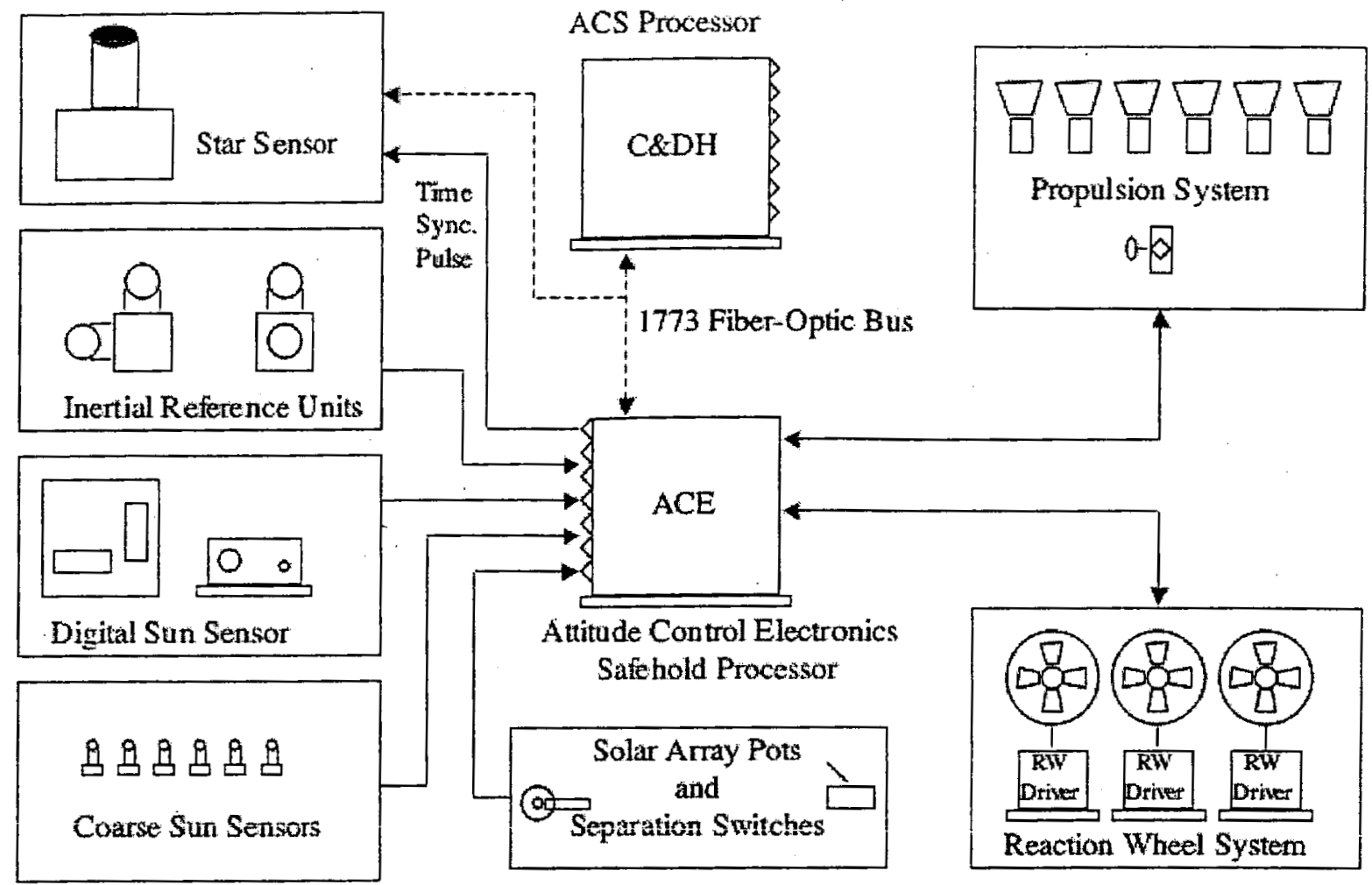

Figure 5: Attitude Control System Architecture

The IRU comprises two Kearfott Two-Axis Rate Assemblies (TARAs), one with input axes aligned with the $\mathbf{z}_{B}$ and $\mathbf{x}_{B}$ axes and the other with input axes aligned with the $\mathbf{z}_{B}$ and $\mathbf{y}_{B}$ axes. This gives redundant rate inputs on the $\mathbf{z}_{\mathrm{B}}$ axis; the DSS outputs can be differentiated to provide rates on the other axes in the event of an IRU failure.

The boresights of the two Lockheed-Martin ASTs ${ }^{18}$ are in the $\pm \mathbf{y}_{\mathrm{B}}$ directions. Each AST tracks up to 50 stars simultaneously in its $8.8^{\circ}$ square FOV, matches them to stars in an internal star catalog, and computes its attitude as a GCI-referenced quaternion with accuracy of 21 arcseconds $(1 \sigma)$ around its boresight axis and 2.3 arc-seconds $(1 \sigma)$ in the other two axes. 
The Adcole two-axis DSS has two heads, each with $64^{\circ}$ square FOV and an accuracy of 1 arcminute $(3 \sigma)$. The centers of the FOVs of the two heads are in the $\mathbf{x}_{\mathrm{B}}-\mathbf{z}_{\mathrm{B}}$ plane at angles of $\pm 29.5^{\circ}$ from the $\mathbf{z}_{B}$-axis. The CSSs are cosine eyes located in pairs looking outward from the edges of the six solar array panels, alternately pointing $36.9^{\circ}$ up and $36.9^{\circ}$ down from the $\mathbf{x}_{B}-\mathbf{y}_{B}$ plane.

The RWAs are Ithaco Type E wheels each with a momentum storage capacity of $70 \mathrm{Nms}$. The available reaction torque of each wheel is $0.35 \mathrm{Nm}$, but this is limited to $0.215 \mathrm{Nm}$ by the WMAP software to satisfy power constraints. The reaction wheel rotation axes are tilted $60^{\circ}$ from the $-\mathbf{z}_{B}$ axis and uniformly distributed $120^{\circ}$ apart in azimuth about this axis. The wheels serve the dual function of counterbalancing the body's spin angular momentum to maintain the system momentum (i.e. body plus wheels) near zero while simultaneously applying control torques to provide the desired spacecraft attitude. The wheel axis orientations result in all wheel speeds being biased away from zero while the spin-scan observing motion is being executed, thus avoiding zero-speed crossings that would occur if the wheel spin axes were oriented along the spacecraft body frame coordinate axes.

The propulsion system comprises eight monopropellant hydrazine Reaction Engine Modules (REMs) and associated hardware. Each REM generates a maximum thrust of $4.45 \mathrm{~N}$.

\section{MOMENTUM MANAGEMENT STRATEGY}

The choice of an $\mathrm{L}_{2}$ orbit to minimize magnetic, thermal, and radiation disturbances precludes the use of magnetic sensing or torquing. Thus, the propulsion system provided for orbit maneuvers and stationkeeping is also used to unload accumulated system angular momentum after each orbit adjust. These occur several times in the phasing loops but no more than once every three months at $\mathrm{L}_{2}$ to minimize interruptions of science observations. The RWAs can store on the order of $70 \mathrm{Nms}$ of angular momentum in non-spinning modes, and a significant fraction of this along the $z_{B}$ axis while spinning about this axis. While executing the Observing Mode 
spin-scan, however, the transverse momentum storage capacity (i.e. in the $\mathbf{x}_{B}-\mathbf{y}_{B}$ plane) is limited to $3 \mathrm{Nms}$, the amount that can be cycled among the three RWAs at the fast spin rate without adversely affecting attitude control.

Gravity-gradient, atmospheric drag, and outgassing torques are significant in the phasing loops, but the accumulated angular momentum of less than $1 \mathrm{Nms}$ per orbit is easily stored until removal following orbit maneuvers at apogee or perigee. Solar radiation pressure torque is the only significant disturbance torque at $\mathrm{L}_{2}$, and the uniform rotation of the spin axis reduces its average along the $\mathbf{x}_{B}$ and $\mathbf{y}_{B}$ axes by more than two orders of magnitude compared to its instantaneous value. The only potentially troublesome component is a "pinwheel" torque along the $z_{B}$ axis, which might result from an imperfect deployment of the solar array panels. The angular momentum is accumulated in inertial space, so it is clear from Figure 3 that the pinwheel torque at one point in the orbit leads to a transverse angular momentum one-quarter orbit, or 91 days, later. This means that any accumulation of angular momentum from the pinwheel torque of more than about $0.03 \mathrm{Nms}$ per day would require momentum unloading more frequently than desired.

Pre-flight estimates of the pinwheel torque gave angular momentum accumulation ranging from 0.0016 to $0.065 \mathrm{Nms}$ per day, depending on the accuracy of deployment of the solar arrays and the resulting symmetry of the spacecraft. ${ }^{14}$ The worst-case estimate would reach the Observing Mode system angular momentum limit of $3 \mathrm{Nms}$ in 46 days, which is highly undesirable. Flight data indicates an angular momentum accumulation of about $0.006 \mathrm{Nms}$ per day, which easily meets the three-month requirement. In fact, since this is less than $0.03 \mathrm{Nms}$ per day, Figure 3 shows that the pinwheel torque will begin to unload the accumulated angular momentum on the next quarter orbit, so no unloading by the REMs is required at all, in principle. The orbit perturbations at $L_{2}$ have also been well within requirements, so it has been possible to perform stationkeeping and momentum unloading only once every four months, rather than every three months. 


\section{ACS OPERATIONAL MODES}

WMAP has six ACS modes. The Inertial, Observing, Delta V, Delta $\mathrm{H}$, and Sun Acquisition modes are implemented in the main spacecraft (Mongoose V) processor, while the Safehold Mode resides in the Attitude Control Electronics Remote Services Node (ACE RSN). Figure 6 shows the modes and the transitions among them. Anomalous behavior can result in autonomous transitions from any other mode to Sun Acquisition Mode or Safehold Mode, even though these transitions are not shown explicitly. Each of the modes of the WMAP ACS will be

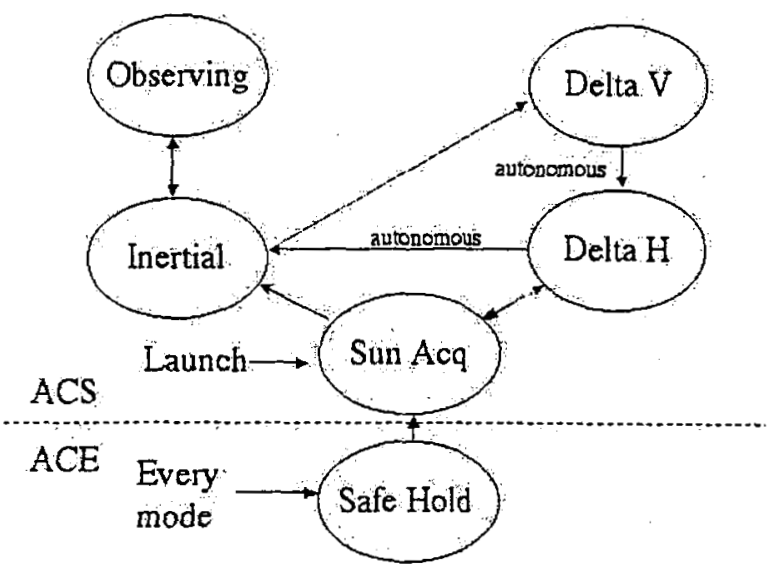
discussed below, including a discussion of the sensors, actuators, and control algorithms used in that mode. Examples of in-flight performance are also provided.

\section{ACS DESIGN AND PERFORMANCE}

\section{Sun Acquisition Mode}

Sun Acquisition Mode uses the CSS, IRU, and RWAs to acquire and maintain the spacecraft $z_{B}$ axis within $25^{\circ}$ of the Sun, starting from any initial orientation and with any initial body momentum less than $[13,13,55]$ Nms. This is a thermally safe and power-positive orientation before instrument power-on, and this is the mode entered after separation from the launch 
vehicle. If the rates at entry to the RWA-based Sun Acquisition Mode exceed those that can be handled by this mode, the REM-based Delta H Mode is entered to reduce the rates to an acceptable level, after which the spacecraft returns to Sun Acquisition Mode. Transition from Sun Acquisition Mode to Inertial Mode can be commanded after the Sun has been acquired. Transition to the Mongoose control modes from the ACE Safehold Mode is through Sun Acquisition Mode. Normal exit from Sun Acquisition Mode is to either Delta H or Inertial Mode, depending on the residual spacecraft spin rate.

The attitude error signals in Sun Acquisition Mode are calculated by computing the cross product of the sun vector computed from CSS measurements with the desired sun vector. The nominal desired sun vector points the $+\mathbf{z}_{B}$ axis, the solar array normal, directly at the sun. The attitude error signals are limited and multiplied by the proportional control gain. The rate error signal is the body rate measured by the IRUs. The rate error vector is multiplied by the spacecraft inertia and then each axis is multiplied by a derivative control gain. The output of this proportionalderivative (PD) control algorithm is three torque commands in the body frame. A body-to-wheel reference frame transformation matrix is used to transform these commands to the reaction wheel frame. The wheel torque commands are scaled down by a common factor if the largest command exceeds the wheel torque capability, so that the torque direction is preserved while the largest command is the maximum reaction wheel torque command.

In pre-launch analysis and testing, Sun Acquisition. Mode was found to meet its performance requirements for all initial system momentum magnitudes of $55 \mathrm{Nms}$ or less. Because this level represented $2 \sigma$ separation rates from the Delta $\Pi$ third stage, a contingency thruster momentum unload was unlikely, but would be possibly needed. In addition, there were a few degenerate cases (e.g., 180 degrees off of the sun with zero rates) that did not satisfy the requirements, but these were deemed too unrealistic to be of concern. 
Performance on orbit was as expected from the pre-launch testing. After a post-launch communications gap, contact was established with WMAP via the Tracking and Data Relay Satellite TDRS-W at 21:03, 77 minutes after launch and 10 minutes prior to spacecraft separation. At the beginning of this contact, WMAP was still in the Delta II third-stage spin; gyro rates were saturated (over $5.3 \% \mathrm{sec}$ ) and the system momentum magnitude measurement was over $90 \mathrm{Nms}$, as shown in Fig. 7. At 21:13, the yo-yo despin from the third-stage spin occurred, and WMAP separated from its booster. Within one telemetry update (16 seconds), the gyros desaturated with all axes below $1 \%$ second. The measured system momentum dropped in that update to approximately $10 \mathrm{Nms}$. The solar arrays began to deploy 14 seconds after separation. The spacecraft reported that the arrays were deployed (all arrays deployed to within $25^{\circ}$ of their fully deployed state) 8 seconds later. The inset in Figure 7 shows the change in the system momentum magnitude measurement as the mass properties of the spacecraft changed during array deployment and fuel spin down. The arrays opened fully in four minutes, at which point the system momentum magnitude was $7 \mathrm{Nms-well} \mathrm{within} \mathrm{the} \mathrm{maximum} \mathrm{level} \mathrm{of} 55 \mathrm{Nms}$ at which Sun Acquisition Mode could acquire the sun.

Figure 8 shows the CSS and DSS measured sun angles at separation. Note that the sun was out of the DSS field of view until 5530 seconds of the plot. The spacecraft acquired the sun within 7 minutes of separation. The spacecraft was declared separated and safe on the sun at $21: 26,13$ minutes after separation. 


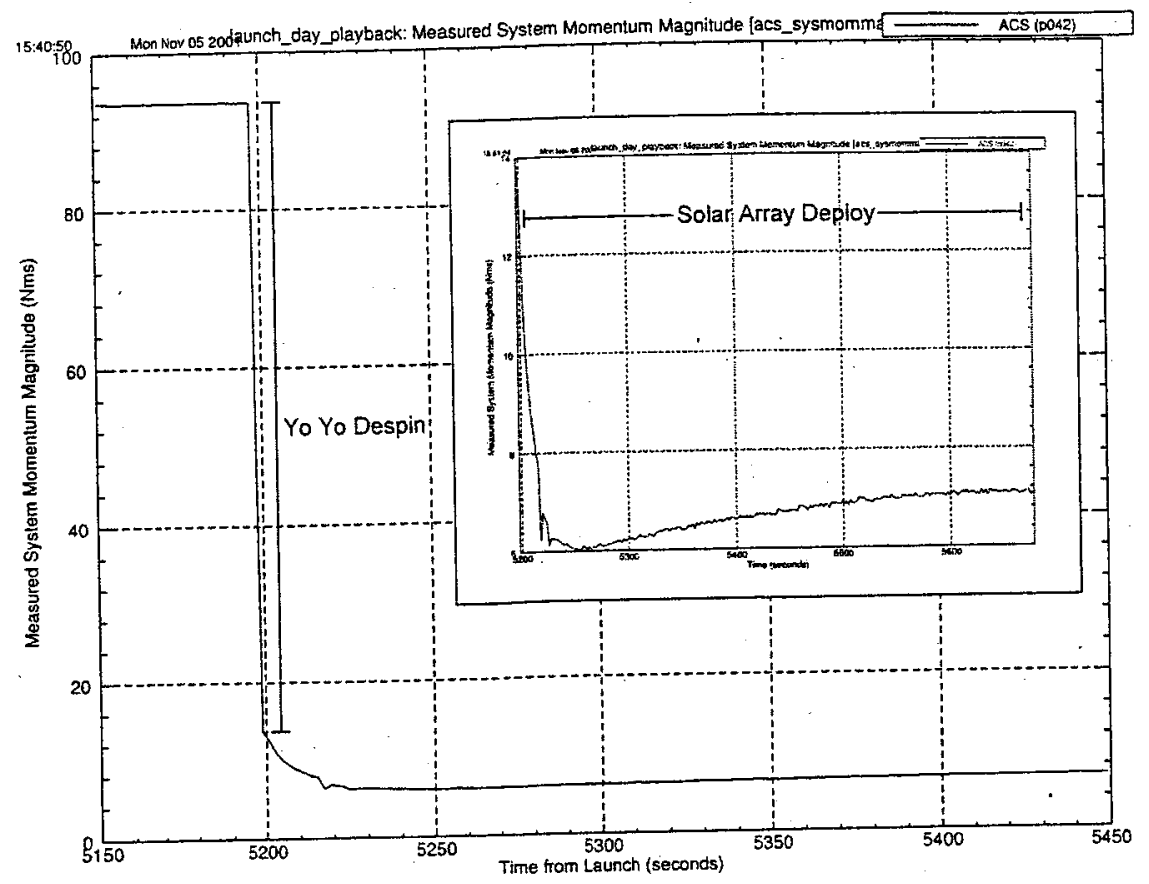

Figure 7: System Momentum Magnitude at Separation

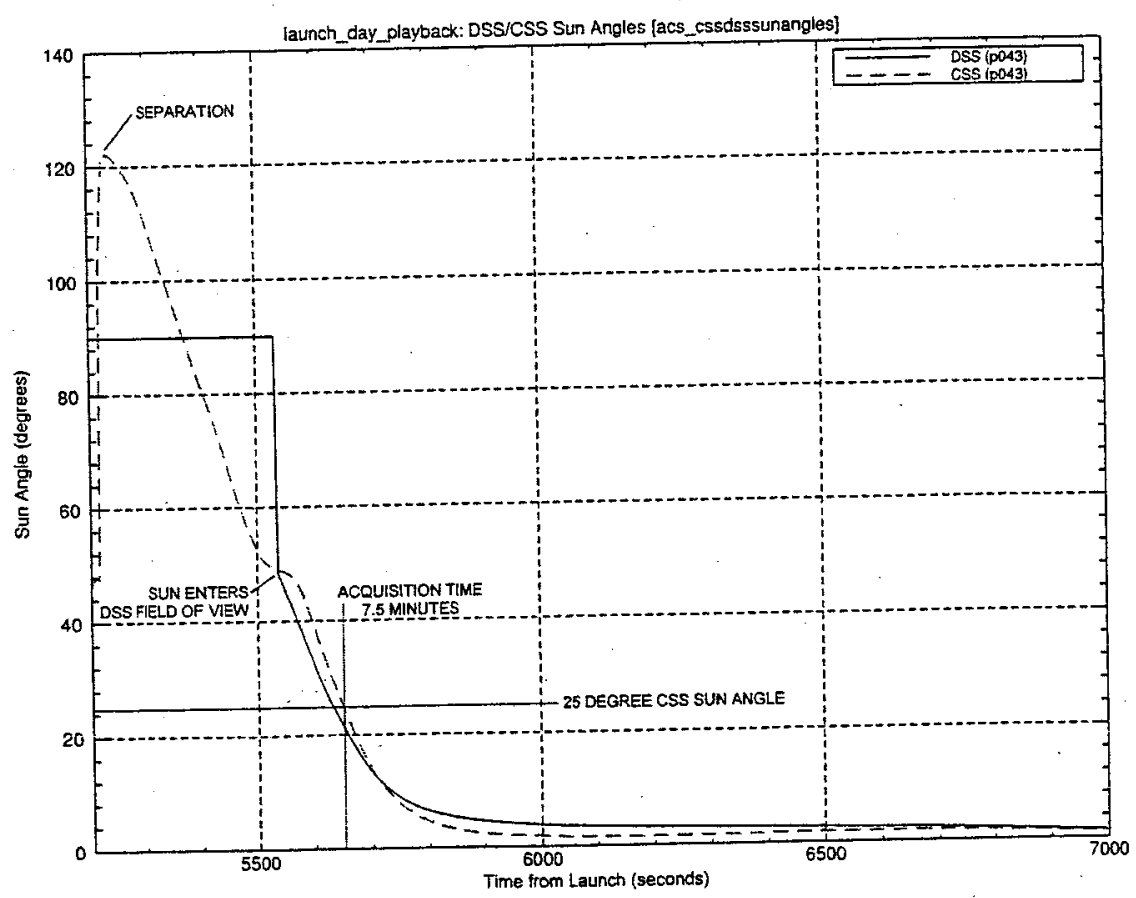

Figure 8: Sun Angles at Separation 
In the design of the Failure Detection and Correction logic for WMAP, Sun Acquisition Mode was used in many cases as a first level of safing in the event of a problem. Fortunately, there were no on-orbit anomalies that caused an autonomous entrance into Sun Acquisition Mode. During the first week of the launch and in-orbit checkout period, Sun Acquisition Mode was used as the "base" mode to keep the spacecraft in when other operations were not being conducted. Once Observing Mode was checked out, it or Inertial Mode became the base mode. The maneuver plan used for each of the calibration maneuvers at apogee and orbit maneuvers at perigee commanded the spacecraft into Sun Acquisition immediately after the burn. In all cases, the mode satisfied all of its requirements.

\section{Inertial Mode}

Inertial Mode acts as a staging mode between the other operations of the spacecraft; all Mongoose modes other than Delta $\mathrm{V}$ have the capability to enter this mode. Inertial Mode can either hold the spacecraft in an inertially-fixed orientation or slew the spacecraft between two different orientations. This is an RWA- and IRU-based mode, with DSS and AST measurements used in an onboard Extended Kalman Filter to update the gyro bias and quaternion error estimates. Normal exits from Inertial Mode are by ground command only. The high-level requirements for Inertial Mode are to acquire and hold a fixed target quaternion within 10 minutes and to maintain the spacecraft $\mathbf{z}_{\mathrm{B}}$ axis within $25^{\circ}$ of the sunline at all times. The

desired orientation is commanded as a desired GCI-to-body quaternion $q_{\mathrm{c}^{\cdot}}{ }^{20,21}$ A slew will be executed if this is not close to the current spacecraft orientation. The attitude control is by RWA torques, which are computed by a PD controller in terms of attitude and rate errors.

The attitude errors are expressed as twice the vector part $\mathbf{q}_{\mathrm{e}}$ of an error quaternion, which is the quotient of the commanded quaternion and an estimated quaternion $\hat{q}$ :

$$
q_{e}= \pm q_{c} \otimes \hat{q}^{-1}
$$


In this and the following we use the quaternion product convention of Refs. 21 and 22 rather than that of Ref. 20 so that the order of quaternion multiplication is the same as that of the corresponding direction cosine matrices. The sign in the equation above is chosen to assign a positive sign to the scalar component of $q_{\mathrm{e}}$, which has magnitude close to unity for small pointing errors. The small angle approximation is used at all times for the attitude error; this introduces inaccuracies for large slews, but does not affect the performance of the controller, and as there is not direction preference for the slews, it is not a problem.

A dynamic attitude limiter is employed in Inertial Mode to enable the spacecraft to meet the $25^{\circ}$ sun constraint during slews. Before implementing the limiter, the sun line angle reached as high as $38^{\circ}$ in simulated $45^{\circ}$ Inertial Mode slews including high spin errors in the $\mathbf{z}_{\mathrm{B}}$ axis,. The dynamic attitude error limiter calculates an attitude error limit for each axis proportional to the error in that axis. This preserves the direction of the resulting slew and prevents the spacecraft from violating the sun constraint for any slews with spin angles from $0^{\circ}$ to $180^{\circ}$.

The estimated quaternion is computed by the Kalman filter, with IRU, AST, and DSS measurements as input. ${ }^{22-24}$ This is similar to Kalman filters employed on several previous Goddard Space Flight Center missions, except that the AST produces a measured attitude quaternion rather than observed star vectors. This simplifies the onboard software computations by removing the burden of star identification and the necessity to carry an onboard star catalog. ${ }^{18}$ The Kalman filter will be discussed in more detail later in this paper.

The onboard Kalman filter also estimates corrections to the gyro drift rates that are subtracted from the IRU-measured body rates to produce the body rate vector. Since the desired rates in Inertial Mode are zero, the rate error vector $\omega_{e}$ is the negative of the body rate vector measured by the IRU:

$$
\omega_{\mathrm{e}}=-\omega_{\mathrm{BI}}
$$


The commanded control torque in the body coordinate system is given by: $:^{24-26}$

$$
\mathbf{T}=J\left[k_{\mathrm{d}} \boldsymbol{\omega}_{\mathrm{e}}+k_{\mathrm{p}}\left(2 \mathbf{q}_{\mathrm{e}}\right)+k_{\mathrm{i}} \int\left(2 \mathbf{q}_{\mathrm{e}}\right)\right]
$$

where $J$ is the WMAP moment of inertia tensor, and $k_{\mathrm{d}}, k_{\mathrm{p}}$, and $k_{\mathrm{i}}$ are the derivative, proportional, and integral gains, respectively ( $k_{\mathrm{i}}$ is nominally zero). The factors of two multiplying the error quaternion and its integral reflect the fact that the quaternion errors are half the angle errors in the small angle approximation. The controller outputs are the same type of torque commands as described in the Sun Acquisition section, and are sent to the RWAs in the same manner.

In addition to accepting ground-commanded attitudes, Inertial Mode has two "precanned" quaternions onboard. One will point the spacecraft $+\mathbf{z}_{\mathrm{B}}$ axis towards the sun, and the other will point the spacecraft $+z_{B}$ axis $22.5^{\circ}$ from the sun. The commands have fixed quaternions relative to the RSR frame described previously. The RSR frame orientation relative to the GCI frame is calculated as a quatemion every minute onboard, and this quaternion is multiplied by the stored quaternion at the command time to yield the commanded body attitude relative to the GCI frame.

Inertial Mode also accepts quaternions from a Command Quaternion Table (CQT), which is a table of times and quaternions representing a particular attitude profile for the duration of the CQT. The CQT can be loaded with different quaternion sequences, and was used to command the gyro calibration slew profile as part of the in-orbit checkout. The CQT was also used to command the spacecraft to the proper burn orientations, and to command the spacecraft to hold the thrust direction parallel to the velocity vector before, during, and after the maneuvers.

WMAP was in Inertial Mode for a long period of time during the first week of the mission, leading to one surprise: Inertial Mode does not hold the spacecraft in an inertially fixed orientation. When the spacecraft was commanded to point at the current sun location (via the sun pointing precanned command discussed above), the sunline angle from the spacecraft $\mathbf{z}_{B}$ axis was expected to change by about one degree per day, consistent with the spacecraft's motion relative 
to the sun. Instead, the spacecraft telemetry showed that the spacecraft maintained a sun-pointing attitude. The difference between the estimated quaternion and the command quaternion slowly climbed at approximately $1 \mathrm{deg} / \mathrm{day}$, and was "reset" to zero whenever we commanded a sunpointing attitude, as expected. However, the telemetered attitude errors and sun angles did not reflect this. Inspection of the flight code and ACS simulation code revealed that the portion of the simulation used to automatically generate flight software only understands commands and attitudes in the RSR frame. When a new inertial (GCI) quaternion is commanded, the flight software converts it to an RSR reference frame quaternion before handing it off to the controller. If this conversion were done each time the controller was entered (i.e., once per second) or when the ephemeris was updated, then the command quaternion within the Inertial Mode controller would remain inertially fixed. However, this conversion is only done with the initial command, so the controller continues to control to the static RSR frame quaternion. As the ephemeris Lipdates and the RSR frame moves, the controller moves with the RSR frame. Thus, commanding a sun-pointing attitude results in the Inertial Mode controller continuing to follow the sun. This was not discovered in testing primarily because nominal operations never involved remaining in Inertial Mode for an entire day. The longest stay in Inertial Mode in a simulation run, build test, or acceptance test was an hour or less, so the effect was too small to be seen in the test data.

\section{Observing Mode}

Observing Mode is an IRU-based mode used for science operations, using the same Kalman filter as Inertial Mode. The RWAs provide the angular momentum that preserves a near-zero system momentum and the torques that maintain both the $22.5^{\circ}$ angle between the spin axis and the sunline and the desired sky-scan rates illustrated in Figure 2. The tolerance on both rates is $5 \%$, and the tolerance on the Sun line angle is $0.25^{\circ}$. Observing Mode differs from Inertial Mode in that the commanded quaternion is time-varying, the commanded rates are non-zero, and a commanded acceleration and a commanded gyroscopic torque are used in a feedforward loop to eliminate attitude hangoff. The attitude error is computed as in Inertial Mode, but the 
commanded attitude quaternion comes from the desired motion relative to the sunline, rather than being fixed. Normal transitions into or out of Observing Mode are via Inertial Mode.

The commanded inertial quaternion is computed as the product of an RSR-to-body and a GCI-toRSR quaternion:

$$
q_{\mathrm{BI}}=q_{\mathrm{BR}} \otimes q_{\mathrm{RI}}
$$

The GCI-to-RSR quaternion is computed onboard from ephemeris models. The desired RSR-tobody attitude is more conveniently expressed in terms of 3-1-3 Euler angles ${ }^{20,21}$ than in terms of a quaternion. The commanded values of the three Euler rates are those that give the desired scan rates:

$$
\begin{aligned}
& \dot{\phi}_{\mathrm{c}}=-1 \mathrm{rev} / \mathrm{hour}=-0.001745 \mathrm{rad} / \mathrm{sec} \\
& \dot{\theta}_{\mathrm{c}}=0 \\
& \dot{\psi}_{\mathrm{c}}=0.464 \mathrm{rpm}=0.04859 \mathrm{rad} / \mathrm{sec}
\end{aligned}
$$

The commanded values of the three Euler angles are then given by:

$$
\begin{aligned}
& \phi_{c}=\phi_{0}+\int_{t_{0}}^{t} \dot{\phi}_{c} \mathrm{dt} \\
& \theta_{c}=22.5^{\circ}=0.3927 \mathrm{rad} \\
& \psi_{c}=\psi_{0}+\int^{t} \dot{\psi}_{c} \mathrm{dt}
\end{aligned}
$$

where $\phi_{0}$ and $\psi_{0}$ are set by the initial state and $\theta_{0}$ by the desired Sun angle. These 3-1-3 Euler angles are converted to the commanded RSR-to-body quaternion $q_{\mathrm{BR}}$ by the standard equations..$^{20,21}$

The rate error vector $\omega_{e}$ is the difference between a commanded body rate vector $\boldsymbol{\omega}_{c}$ and the body rate vector $\omega_{\mathrm{BI}}$ measured by the IRU:

$$
\omega_{e}=\omega_{c}-\omega_{B I}
$$


The commanded body rate vector $\omega_{c}$ is computed from the commanded Euler angles and rates by the standard equation: ${ }^{20,21}$

$$
\left.\omega_{c}=\left[\begin{array}{ccc}
\sin \theta_{c} \sin \psi_{c} & \cos \psi_{c} & 0 \\
\sin \theta_{c} \cos \psi_{c} & -\sin \psi_{c} & 0 \\
\cos \theta_{c} & 0 & 1
\end{array}\right] \begin{array}{c}
\dot{\phi}_{c} \\
\dot{\theta}_{c} \\
\dot{\psi}_{c}
\end{array}\right]
$$

This commanded rate is modified in the Observing Mode controller so that a large rate command change when entering or exiting the mode would not cause the spacecraft to violate the sunline constraint. If the rate error is too large, than the rate path saturates the wheel command, and no attitude error signal gets into the control torque. The result is an uncontrolled spacecraft attitude until the rate errors are small. The initial commanded rate is differenced with the previous derived rate, limited, and then integrated to get a new derived rate, as shown in Figure 9. This new commanded rate is used to calculate the rate error described above and the feedforward terms discussed below. The result is a steady increase in the amount of the initial command rate fed into the control system, which generates a spin up (when entering) and a spindown (when exiting) Observing Mode. This effect is seen in Figure 10. This shows the initial slew (starting just after 1000 seconds on the plot) from the $\mathbf{z}_{B}$ axis on the sunline to the $22.5^{\circ}$ sunline angle, and then the spinup into the compound spin, starting at about 1600 seconds on the plot.

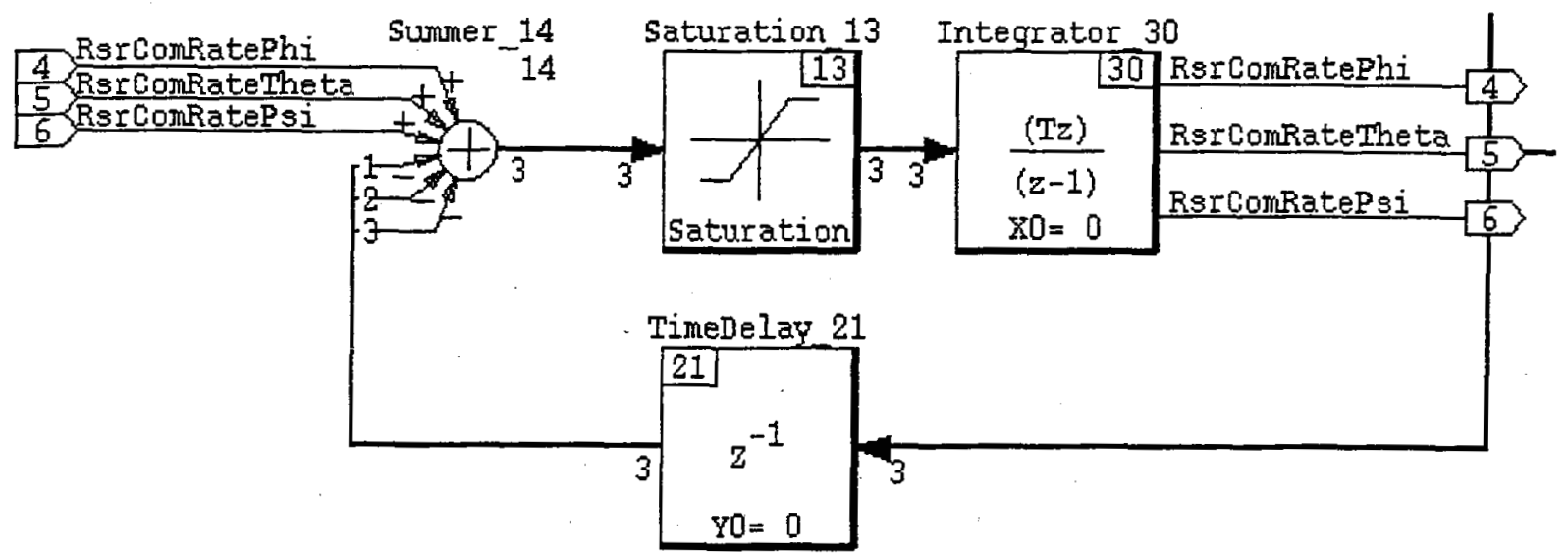

Figure 9: Commanded Rate Limiting 


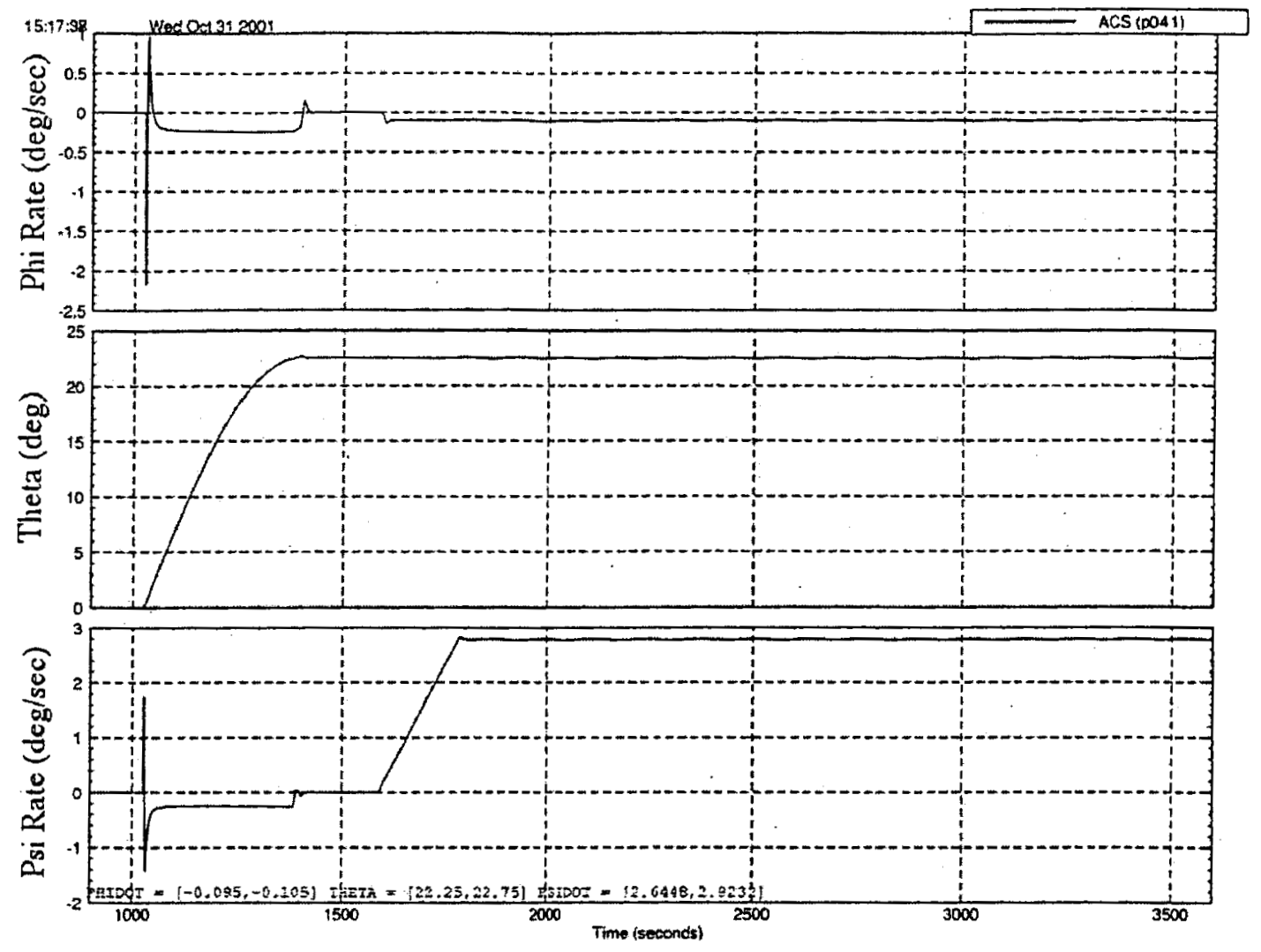

Figure 10: Euler Angles Entering Observing Mode

One of the feedforward terms is the acceleration the spacecraft needs to follow the commanded attitude and rates, and is derived by differentiating the equation for $\omega_{c}$ with respect to time. Since $\dot{\phi}_{c}, \dot{\psi}_{c}$, and $\theta_{c}$ are all constant, the commanded acceleration is:

$$
\dot{\boldsymbol{\omega}}_{\mathrm{c}}=\dot{\psi}_{\mathrm{c}} \dot{\phi}_{\mathrm{c}} \sin \theta_{\mathrm{c}}\left[\begin{array}{c}
\cos \psi_{\mathrm{c}} \\
-\sin \psi_{\mathrm{c}} \\
0
\end{array}\right]
$$

This acceleration is multiplied by the spacecraft inertia to get a commanded torque.

The second feedforward torque is the gyroscopic feedforward torque, used to move the system momentum around among the reaction wheels as required by its constancy in inertial space:

$$
\mathrm{T}_{\mathrm{g}}=\omega_{\mathrm{c}} \times \mathbf{H}_{\mathrm{sys}}
$$


These feedforward torques are added to the output of the PD controller to generate the complete control torque for the RWAs:

$$
\mathbf{T}=J\left[k_{\mathrm{d}} \boldsymbol{\omega}_{\mathrm{e}}+k_{\mathrm{p}}\left(2 \mathbf{q}_{\mathrm{e}}\right)+k_{\mathrm{i}} \int\left(2 \mathbf{q}_{\mathrm{e}}\right)+\dot{\boldsymbol{\omega}}_{\mathrm{c}}\right]+\mathbf{T}_{\mathrm{g}}
$$

This control torque is in the body frame, and is treated the same as the control torque created in Inertial Mode and Sun Acquisition Mode.

Initially, the precession rate $\dot{\phi}$ in Observing Mode did not meet its 5\% accuracy requirement, showing a $7 \%$ variation at the spin period. This was attributed to an inaccurate value of system momentum in the gyroscopic feedforward loop arising from a scale factor error in the RWA tachometer signals. Evidence for this was that the magnitude of the system momentum, which should be constant, had a $0.4 \mathrm{Nms}$ oscillation at spin period and increased during spin-up by 1.0 Nms. Comparing a high-fidelity simulation with flight data determined that the oscillation and spin-up offset could be removed by a small change in the tachometer scale factors by about $2.5 \%$ for RWA1 and about $4 \%$ for RWA2 and RWA3. After loading these new scale factors, the variation of the precession rate was dramatically reduced, as were the spin-period oscillation and the spin-up offset in the computed system momentum magnitude shown in Figure 11. A recent analysis is in basic agreement with this analysis. ${ }^{27}$

Once all sensors and actuators were calibrated, Observing Mode met the 5\% requirement on the commanded Euler rates and the $0.25^{\circ}$ sun angle control requirement, as shown in the time plots in Figure 12 and the nearly perfect circle described by the sun sensor data in the body frame displayed in Figure 13. 

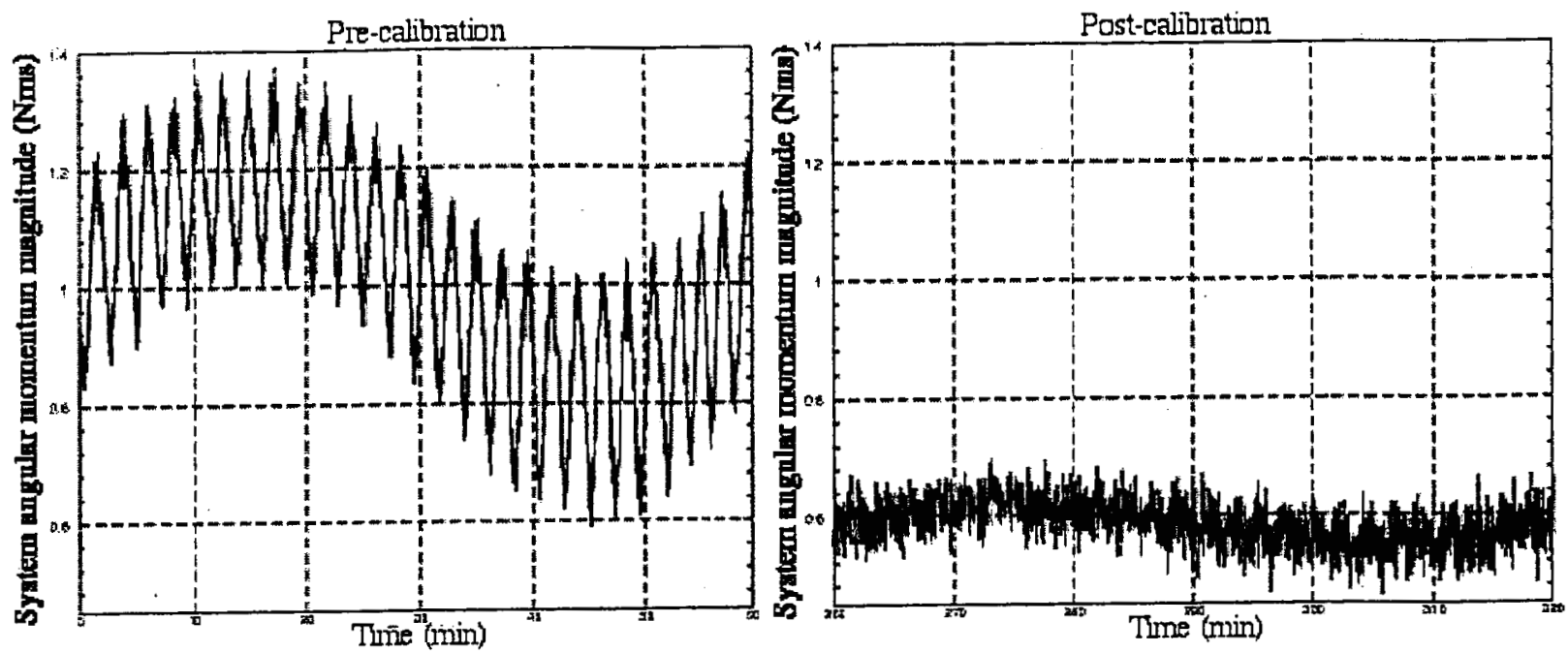

Figure 11: Pre- and Post-Calibration System Angular Momentum Magnitude

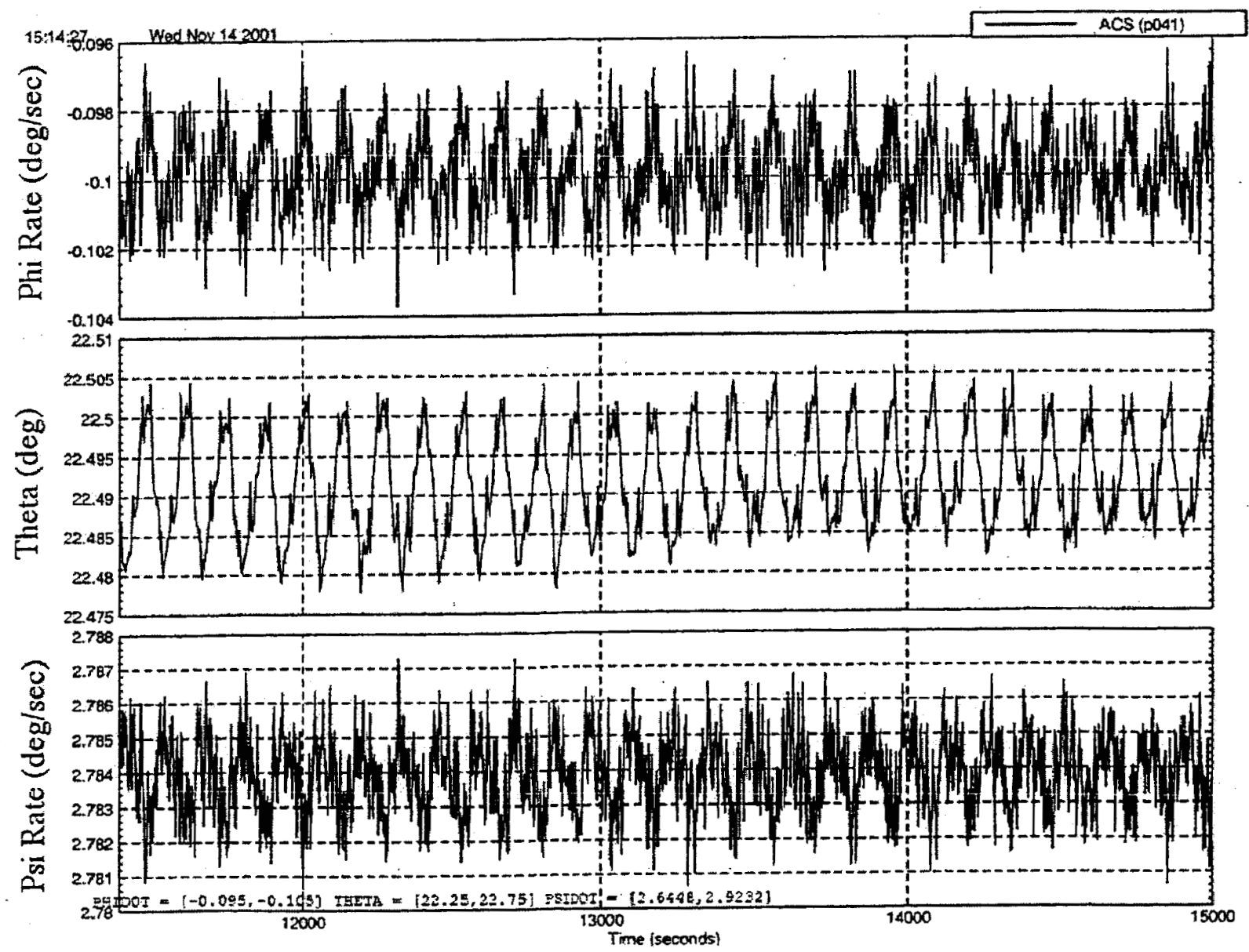

Figure 12: Post-Calibration Observing Mode Performance 


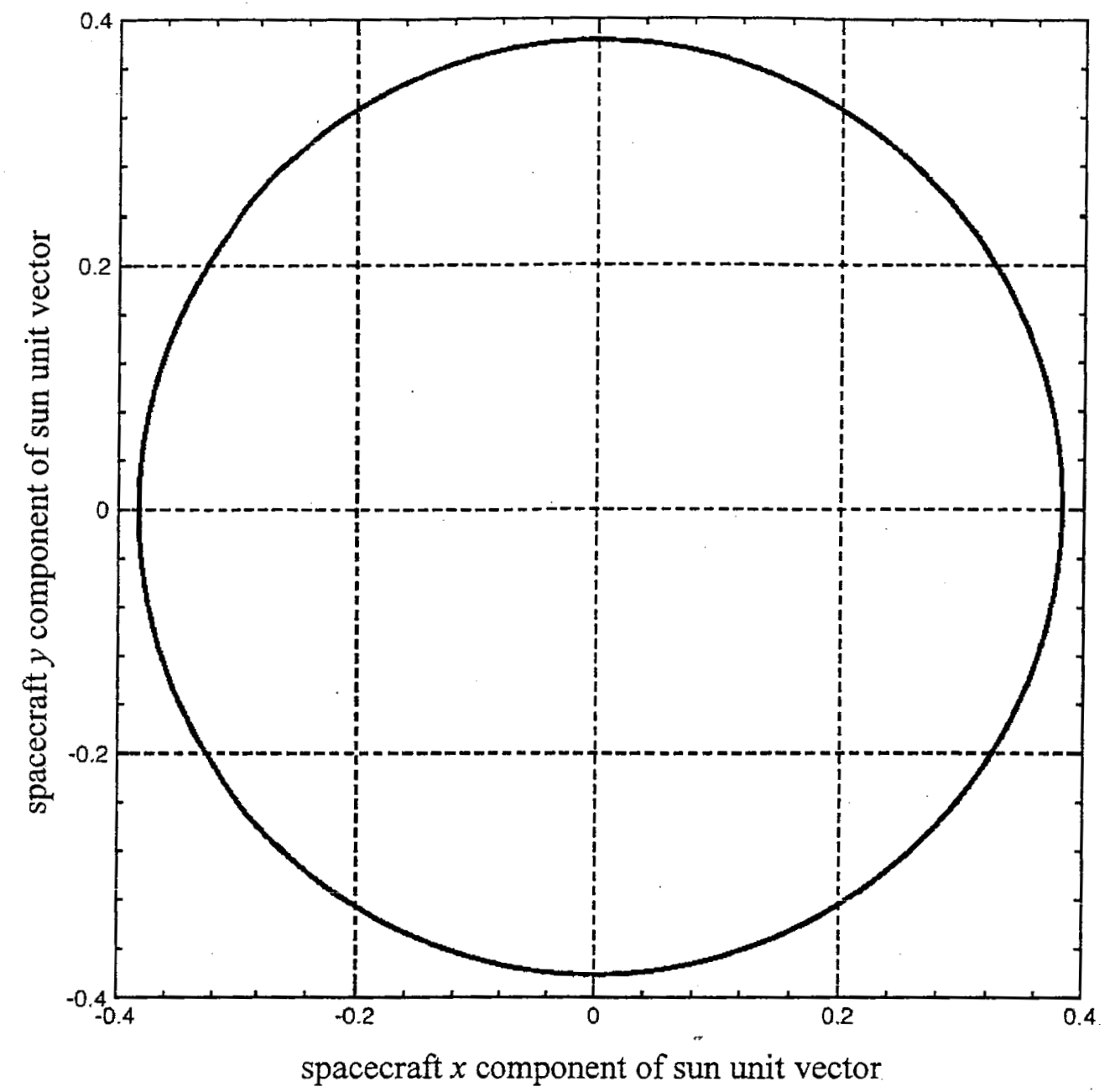

Figure 13: DSS Measurements in Observing Mode

\section{Delta V Mode}

Delta V Mode, which uses the REMs to adjust the orbit in either the initial phasing loops or for $\mathrm{L}_{2}$ stationkeeping, is only entered from Inertial Mode by a command sequence specifying burn duration, direction and start time. The desired attitude in terms of either a single quaternion or a CQT can be configured either via command or by table load. The desired set of thrusters to be used is specified via command. The spacecraft remains in Inertial Mode to slew from the initial orientation to the desired attitude for the start of the maneuver, and transitions to Delta V Mode at the start time of the requested burn. The only sensors used in Delta V Mode are the IRU and RWA tachometers. This mode uses a PD controller to hold the spacecraft to a commanded 
quaternion attitude while executing the Delta V burn. The output of the controller is transformed into thruster firing commands using a pulse width modulator with a minimum pulse width of $0.04 \mathrm{sec}$. The desired attitude is held by off-pulsing the primary set of thrusters and on-pulsing the others. Normal exit is autonomously to Delta H Mode.

Anomalous spacecraft motion experienced during some early maneuvers was later attributed to torques caused by the evaporation of ice condensed on the back of the solar arrays and sun shields. ${ }^{28}$ Despite this unexpected phenomenon, maximum pointing errors during the nine Delta $\mathrm{V}$ maneuvers performed in the first three months of the mission were smaller than predicted $\left(3.7^{\circ}\right.$ vs. $5.5^{\circ}$ ), and the imparted velocity increments were accurate to $1 \%$. Less than $15 \mathrm{~kg}$ of hydrazine propulsion fuel was expended to get to $L_{2}$, about half the amount budgeted for this phase of the mission. The $57 \mathrm{~kg}$ of fuel remaining for stationkeeping and momentum unloading at $\mathrm{L}_{2}$ will easily support a four-year extended mission.

\section{Delta H Mode}

Delta $\mathrm{H}$ Mode uses the REMs to unload spacecraft system angular momentum, which is computed using the RWA tachometers and IRU. It is used primarily upon exit from Delta V Mode, but can be commanded from Inertial or Sun Acquisition Mode if necessary, although this is not anticipated. The same pulse width modulator is used for Delta $\mathrm{H}$ as for Delta V, with the exception that all thrusters are operated in an on-pulsing manner for Delta $H$. If entry was from Delta $\mathrm{V}$ or Inertial Mode, the ACS autonomously transitions to Inertial Mode after the momentum has been reduced to less than $0.3 \mathrm{Nms}$. If Delta $\mathrm{H}$ Mode was entered from Sun Acquisition Mode, as discussed above, the autonomous exit upon completion of the momentum unloading is back to Sun Acquisition Mode. 


\section{Safehold Mode}

Safehold Mode is implemented in the ACE, so it can be entered autonomously in the event of a Mongoose anomaly. It has two configurations, which differ by the rate information used. The first, Safehold/IRU, is a copy of the Sun Acquisition Mode in the Mongoose. The second, Safehold/CSS, is a minimum-hardware mode using only the RWAs and CSSs, with rate errors being computed by numerically differentiating the position error signals. Because it lacks body $z$ rate information from the gyros, Safehold/CSS can tolerate less system momentum than can Sun Acquisition or Safehold/IRU Mode. Since the CSSs are insensitive to rotations about the Sun line, anti-runaway compensation is applied to prevent the wheels from uncontrolled spinning about the satellite's $\mathbf{z}_{\mathrm{B}}$-axis. This is accomplished by applying equal damping torques to the three wheels if the sum of their speeds exceeds a pre-set value, thereby suppressing $\mathbf{z}_{\mathrm{B}}$-axis rotation without applying a net torque in the $\mathbf{x}_{B}-\mathbf{y}_{B}$ plane. Exit from either Safehold Mode is by ground command only.

Charged particle flux from extreme solar activity on November 5, 2001 caused a power-on reset of the Mongoose processor. The ACS transitioned autonomously to Safehold Mode in the ACE, which functioned exactly as designed to keep WMAP safe. The transition to Safehold Mode was discovered by operations staff at the next telemetry pass about 12 hours later, and recovery to Observing Mode was accomplished within three hours of this discovery.

\section{ATTITUDE DETERMINATION KALMAN FILTER}

\section{Implementation and Operation}

The WMAP Kalman filter is an Extended Kalman Filter that uses AST and DSS measurements to update the IRU-propagated attitude and to update the estimated IRU drift rate. The Kalman filter operates in Sun Acquisition or Safehold Modes, but the spacecraft does not use its output for attitude knowledge in those modes, which control the spacecraft based on an attitude 
calculated from the CSS measurements. Sun Acquisition Mode is thus ideal for observing the Kalman filter performance before using its output to control the spacecraft. The first time the Kalman filter was enabled in Sun Acquisition Mode, parameters such as the measurement noise covariance matrix, the initial covariance matrix, and the residual tolerances were increased to launch values, since the sensors had not been calibrated in flight yet (Table 1). The larger initial parameter values allowed the filter to converge and estimate attitude corrections and IRU bias, albeit at less accuracy than possible with a fully calibrated system.

Table 1: Kalman Filter Noise Parameters

\begin{tabular}{|l|l|l|}
\hline \multicolumn{1}{|c|}{ Noise parameter } & Launch Value & Nominal Value \\
\hline DSS measurement & & \\
\hline AST measurement & $0.5 \mathrm{deg}$ & $0.2 \mathrm{deg}$ \\
\hline & $120 \mathrm{asec}$ & $30 \mathrm{asec}$ \\
\hline Gyro rate random walk & & \\
\hline & $0.01296 \mathrm{asec} / \mathrm{s}^{3 / 2}$ & $0.001296 \mathrm{asec} / \mathrm{s}^{3 / 2}$ \\
\hline Gyro rate white noise & & \\
\hline Initial covariance matrix diagonal & $\left(2^{\circ}\right)^{2},\left(40^{\circ} / \mathrm{hr}\right)^{2}$ & $\left(0.5^{\circ}\right)^{2},\left(20^{\circ} / \mathrm{hr}\right)^{2}$ \\
\hline
\end{tabular}

During each control cycle, the IRU, DSS, and AST data are sampled at slightly different (but deterministic) times. The previous control cycle's estimated attitude quaternion is propagated to the current sample cycle time with a first-order propagator ${ }^{20}$ using the IRU-measured rates, corrected with the previous cycle's gyro bias estimate. The attitude control is executed based on that propagated attitude and measured rate. The DSS and AST data are processed in the sensor data processing algorithms, and the resulting measurements (sun vector and attitude quaternion) are back-propagated to the IRU sample time with another first-order propagator, using the current body rate measurement. The back propagation removes any attitude error that may result from the timing differences between the sensor samples, especially at the nominal Observing 
Mode spin rate. The Kalman filter uses the synchronized measurements to update the propagated quaternion, the estimated gyro drift bias, and the propagated covariance matrix.

Submodes within each control mode are used to determine whether or not the filter will update the attitude and gyro bias estimate. In general, if the control mode produces accelerations on the body, the Kalman filter is set to propagate the covariance matrix, and the estimated attitude and gyro bias are not updated. In Inertial Hold State and Observing Scan/Hold State, the Kalman filter is used to update the estimates.

\section{Attitude Estimate}

The measurement residuals, the difference between the measured quantity and the expected (modeled) quantity, are a good indication of how well the Kalman filter is estimating the spacecraft attitude. Figures 14 and 15 show a typical one-hour period in Observing Mode at $\mathrm{L}_{2}$ for the DSS and the AST, respectively. Much of the DSS residual is dominated by noise from the measurement, but all axes show some spin-period frequency oscillations. The short-period oscillations are due to inaccuracies in the first-order attitude propagator used onboard (discussed below), and the longer period oscillations at the precession period are due to small remaining misalignments between the AST and the DSS.

Figure 15 shows the most striking pattern in the AST $\mathbf{x}_{\mathrm{B}}$-axis residuals. Good measurement accuracy is indicated by the small amount of noise present (a few arcseconds, as in the $\mathbf{z}_{B}$ axis), but periodic oscillations with magnitude \pm 30 arcseconds are quite apparent. There is also a large 15-arcsecond jump near each peak. These oscillations and jumps are about $90^{\circ}$ out of phase with the $\mathbf{y}_{\mathrm{B}}$-axis pattern. In fact, the large jumps coincide with the zero crossing of the spacecraft body rate in each axis. Since the body rates are smooth and continuous, the error is probably in the attitude propagated from that rate signal. The first-order numerical integrator used to propagate the quaternion may induce errors on the order of 15 arcseconds when the rate signal crosses zero. Since the $\mathbf{z}_{B}$ component of the angular velocity is always non-zero, there are no large jumps in 
the $\mathbf{Z}_{\mathrm{B}}$-axis residual. So, the spin-period dependent oscillations are most likely more a reflection of attitude propagation errors than of actual measurement errors.

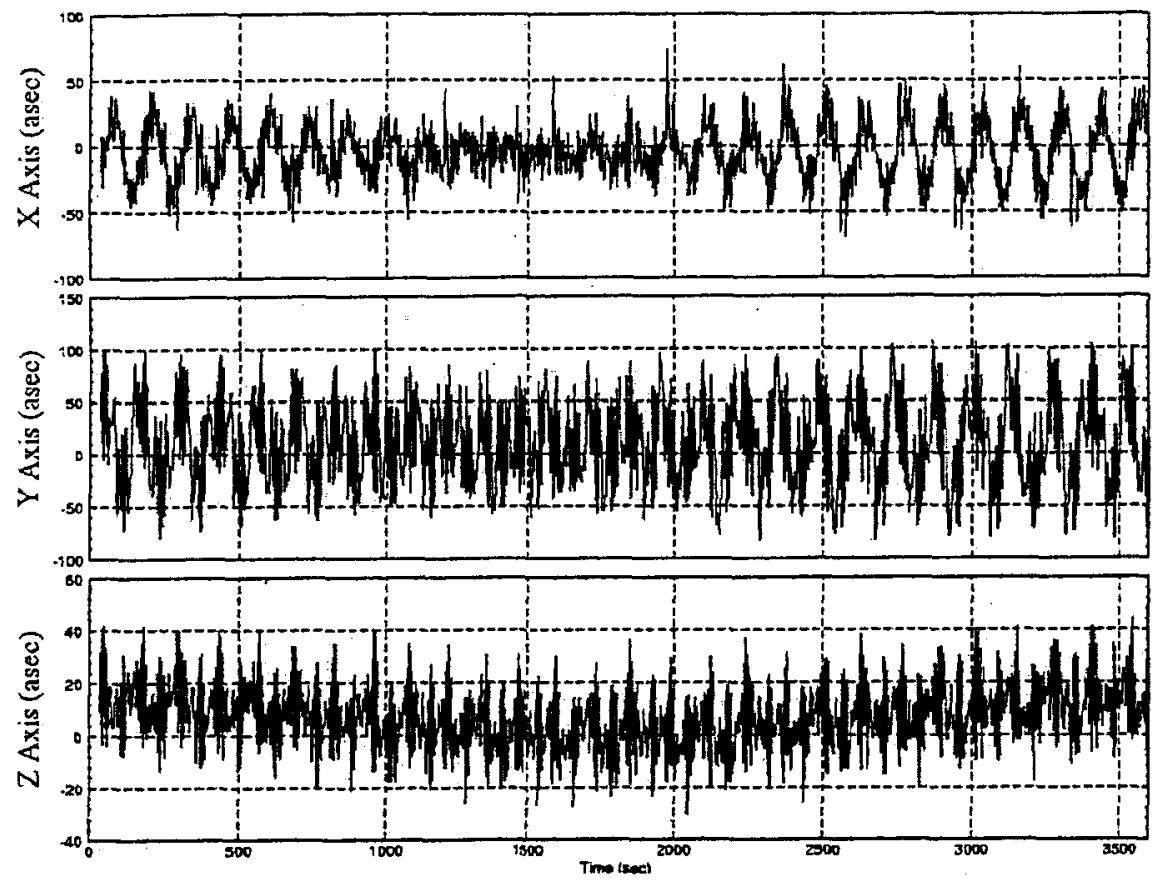

Figure 14: DSS Residuals

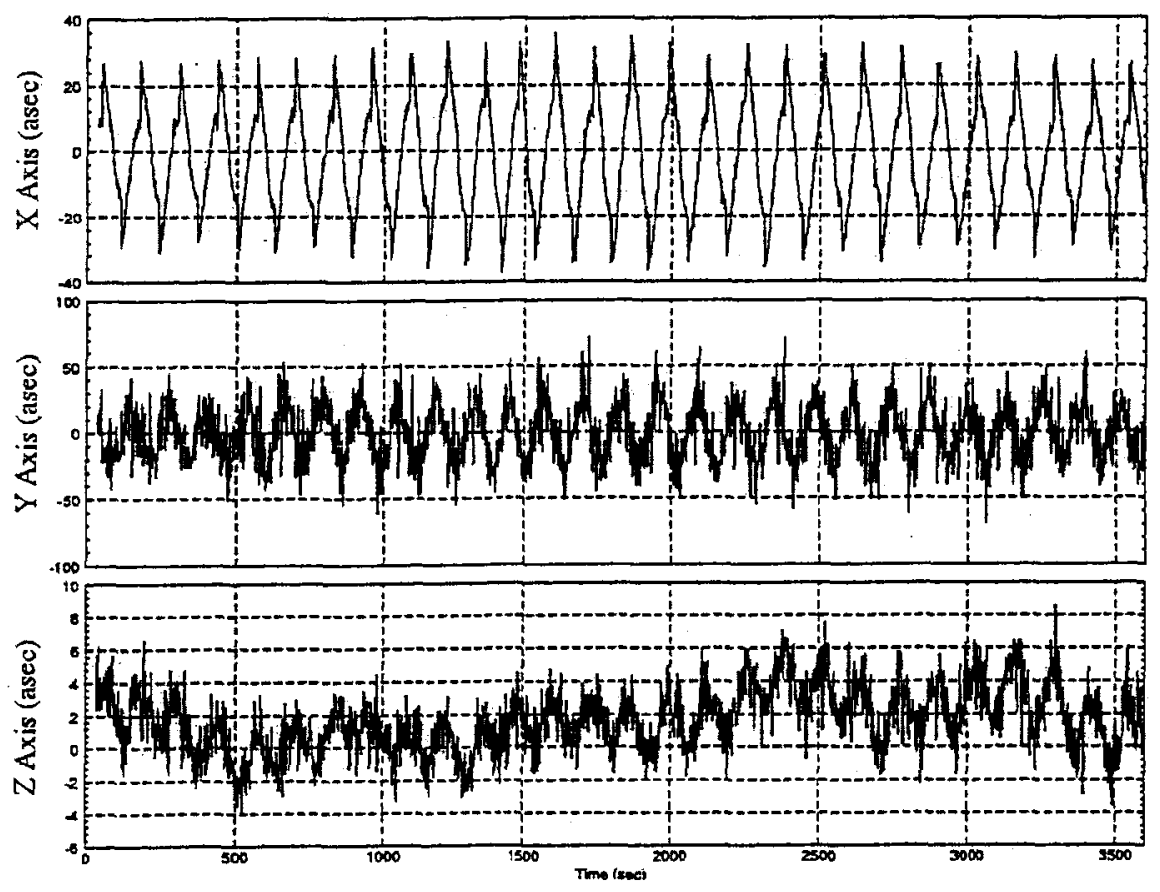

Figure 15: AST Residuals 
In addition, the AST boresight is along the spacecraft $\mathrm{y}_{\mathrm{B}}$ axis, so boresight inaccuracies show up as larger, noisier $y_{B}$-axis AST residuals. The 30 arcsecond magnitude oscillations seen in the $x_{B}$ and $y_{B}$ axis residuals of both the DSS and AST residuals is probably due to the inaccuracy of the first-order propagators used in processing the data and propagating the attitude. The spikes correspond to the zero crossings of the $\mathrm{x}_{\mathrm{B}}$ and $\mathrm{y}_{\mathrm{B}}$ body rates. The $\mathrm{z}_{\mathrm{B}}$ axis has a relatively constant, nonzero rate, so propagation errors effects don't affect the residuals, although all residual components show some spin-period frequency oscillation.

Stray light in the ASTs caused some problems during the phasing loops. Both ASTs lost track when the Moon was within a degree or so of the FOV, but only for a few seconds in a spin cycle, and only for three spin cycles in any precession cycle. No more than 13 AST readings were lost in a precession cycle. There is no Moon, Earth, or Sun interference at $L_{2}$, and the ASTs have been routinely tracking 15 to 40 stars in the absence of interference. Based on the definitive ground attitude solution, the WMAP attitude determination accuracy (RSS Kalman filter error and systematic error) is 1.1 arcseconds ( $x$ and $z$, perpendicular to the AST boresight), and 5.3 arcseconds $(y$, around boresight), all $1 \sigma$. This easily meets the pointing knowledge requirement of 1.3 arcminutes per axis $(1 \sigma)$.

\section{Drift Bias Estimate}

WMAP was launched with default gyro drift bias values of $0 \mathrm{deg} / \mathrm{hr}$ in each axis loaded in a flight software table. These were used to remove the expected gyro drift bias during IRU sensor data processing. The Kalman filter estimates corrections to these table values, and that correction, the estimated bias, is removed from the IRU rates as well. After the filter was first enabled and converged the day after launch, the estimated drift rates were added to the default drift rates and loaded into the IRU table onboard. The filter was reset so that it would only have to estimate small corrections to the new table drift rates. The total gyro biases have remained stable since launch, indicating good gyro health. In fact, the current estimates for the total gyro 
biases (default table values plus current Kalman filter estimate), are $[-10.9,1.4,7.3] \mathrm{deg} / \mathrm{hour}$, and are within $0.25 \mathrm{deg} /$ hour of the total estimates that were loaded the day after launch. The oscillations seen in the $\mathbf{x}_{B}$ axis and $\mathbf{y}_{B}$ axis Kalman filter estimates and the larger bias seen in the $\mathbf{z}_{\mathrm{B}}$ axis estimate (shown in Figure 16) suggest a small error in the IRU scale factor, rather than an actual variation of the sensor data.

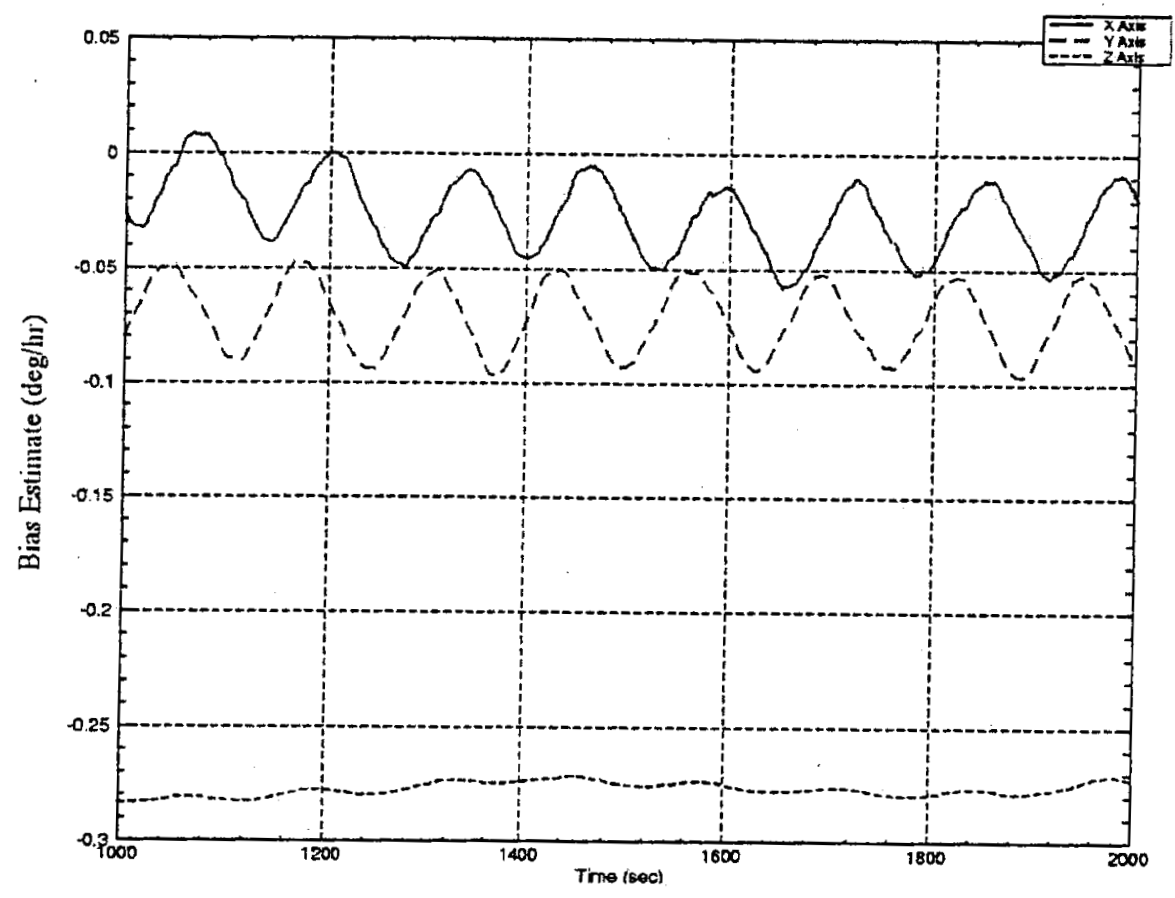

Figure 16: Kalman Filter Estimates of Gyro Bias

\section{CONCLUSIONS}

The WMAP spacecraft has to meet stringent requirements on attitude determination and control while acquiring the sun, slewing, and performing the compound spin necessary to meet the mission science objectives. The attitude control system described in this paper has successfully met these demanding requirements far from the Earth where no magnetic field is useful for sensing or actuation, and with infrequent telemetry passes. The processor upset on November 5 , 2001 illustrated the importance of having a safemode control capability that is independent of the 
primary control hardware and software. The flight results show that the WMAP Attitude Control System meets or exceeds every requirement.

\section{REFERENCES}

1. Boggess, N. W., et al., "The COBE Mission: Its Design and Performance Two Years After Launch," Astrophysical Journal, Vol. 397, No.2, pp. 420-429, 1992

2. Gulkis, S., Lubin, P. M., Meyer, S. S., and Silverberg, R. F., "The Cosmic Background Explorer," Scientific American, Vol. 262, No. 1, pp. 132-139, 1990

3. Smoot, G. F., et al., "Structure in the COBE Differential Microwave Radiometer First-Year Maps," Astrophysical Journal Letters, Vol. 396, pp. L1-L5, 1992

4. Bennett, C.L., et al., "Four-Year COBE DMR Cosmic Microwave Background Observations: Maps and Basic Results," Astrophysical Journal Letters, Vol. 464, pp. L1-L4, 1996.

5. "First-Year Results from WMAP," Astrophysical Journal Supplement Series, Vol. 148, pp. $1-241$

6. Hu, W., Sugiyama, N., and Silk, J., "The Physics of Microwave Background Anisotropies," Nature, Vol. 386, No. 6620, pp. 37-43, 1997

7. Richon, K. V., and Mathews, M. W., "An Overview of the Microwave Anisotropy Probe (MAP) Trajectory Design," Astrodynamics 1997, Volume 97, Advances in the Astronautical Sciences, edited by F. R. Hoots, B. Kaufman, P. J. Cefola, and D. B. Spencer, San Diego, CA, Univelt, Inc., pp. 1979-1998, 1998

8. Cuevas, O., Kraft-Newman, L., Mesarch, M. and Woodard, M., "An Overview of Trajectory Design Operations for the Microwave Anisotropy Probe Mission," 2002 AIAA/AAS Astyrodynamics Specialist Conference, AIAA Paper 2002-4425, Monterey, CA, 2002 
9. Mesarch, M., Rohrbaugh, D., and Schiff, C., "Contingency Planning for the Microwave Anisotropy Probe Mission," 2002 AIAA/AAS Astyrodynamics Specialist Conference, AIAA Paper 2002-4426, Monterey, CA, 2002

10. Mesarch, M. and Andrews, S., "The Maneuver Planning Process for the Microwave Anisotropy Probe (MAP) Mission," 2002 AIAA/AAS Astyrodynamics Specialist Conference, AIAA Paper 2002-4427, Monterey, CA, 2002

11. Edery, A., "Earth Shadows and the SEV Angle of MAP's Lissajous Orbit at $\mathrm{L}_{2}, " 2002$ AIAA/AAS Astyrodynamics Specialist Conference, AIAA Paper 2002-4428, Monterey, CA, 2002

12. Rohrbaugh, D., and Schiff, C., "Station-Keeping Approach for the Microwave Anisotropy Probe (MAP)," 2002 ALAA/AAS Astyrodynamics Specialist Conference, AIAA Paper 20024429, Monterey, CA, 2002

13. Andrews, S. F., Campbell, C. E., Ericsson-Jackson, A. J., Markley, F. L., and O'Donnell, J. R., "MAP Attitude Control System Design and Analysis," Flight Mechanics Symposium 1997, NASA CP-3345, Greenbelt, MD, pp. 445-456, 1997

14. Ericsson-Jackson, A. J., Andrews, S. F., O’Donnell, J. R., Jr., and Markley, F. L., "MAP Stability, Design and Analysis," Spaceflight Dynamics 1998, Volume 100, Advances in the Astronautical Sciences, edited by Thomas H. Stengle, San Diego, CA, Univelt, Inc., pp. $955-$ 969,1998

15. Markley, F. L., Andrews, S. F. , O'Donnell, J. R., , Jr., and Ward, D. K. " The Microwave Anisotropy Probe (MAP) Mission," 2002 AIAA Guidance, Navigation and Control Conference, AIAA Paper 2002-4578, Monterey, CA, 2002 
16. Ward, D. K., Davis, G. T., and O'Donnell, J. R. , Jr., "The Microwave Anisotropy Probe Guidance, Navigation, and Control Hardware Suite," 2002 ALAA Guidance, Navigation and Control Conference, AIAA Paper 2002-4579, Monterey, CA, 2002

17. Andrews, S. F. ; and O'Donnell, J. R. , Jr.," MAP Attitude Control System Design and Flight Performance," 2002 AIAA Guidance, Navigation and Control Conference, AIAA Paper 2002-4580, Monterey, CA, 2002

18. VanBezooijen, R. W. H., Anderson, K. A., and Ward, D. K., "Performance of the AST-201 Star Tracker for the Microwave Anisotropy Probe," 2002 ALAA Guidance, Navigation and Control Conference, AIAA Paper 2002-4582, Monterey, CA, 2002

19. O'Donnell, J. R. Jr., Davis, G. T., and Ward, D. K., "Restoring Redundancy to the MAP Propulsion System," 2002 ALAA Guidance, Navigation and Control Conference, AIAA Paper 2002-4583, Monterey, CA, 2002

20. Wertz, J. R., ed., Spacecraft Attitude Determination and Control, Dordrecht, Holland, D. Reidel, 1978

21. Shuster, M. D., "A Survey of Attitude Representations," Journal of the Astronautical Sciences, Vol. 41, No. 4, pp. 439-517, 1993

22. Lefferts, E. J., Markley, F. L., and Shuster, M. D., "Kalman Filtering for Spacecraft Attitude Estimation," Journal of Guidance, Control, and Dynamics, Vol. 5, No. 5, pp. 417-429, 1982

23. Murrell, J. W., "Precision Attitude Determination for Multimission Spacecraft," 1978 AIAA Guidance and Control Conference, AIAA Paper 78-1248, Palo Alto, CA, pp. 70-87, 1978

24.Bauer, F. H., Femiano, M. D., and Mosier, G. E., "Attitude Control System Conceptual Design for the X-ray Timing Explorer," AIAA Paper 92-4334, AIAA Guidance, Navigation, and Control Conference, Hilton Head, SC (1992). 
25. Mortensen, R. E. "A Globally Stable Linear Attitude Regulator," International Joumal of Control, Vol. 8, No.3, p. 297 (1968)

26. Wie, B., and Barba, P. M., "Quaternion Feedback for Spacecraft Large Angle Maneuvers," Journal of Guidance, Control, and Dynamics, Vol. 8, No. 3, pp. 360-365, 1985

27. Psiaki, M., "Estimation of the Parameters of a Spacecraft's Attitude Dynamics Model Using Flight Data," Flight Mechanics Symposium, Goddard Space Flight Center, Greenbelt, MD, October 2003, NASA Conference Publication NASA/CP-2003-212246

28. Starin, S., O'Donnell, J. R. Jr., Ward, D. K., and Wollack, E. "An Anomalous Force on the MAP Spacecraft," 2002 ALAA Guidance, Navigation and Control Conference, AIAA Paper 2002-4581, Monterey, CA, 2002 